International Journal of Engineering \& Technology, $7(2.25)(2018)$ 68-73
International Journal of Engineering \& Technology
SPC
Website: www.sciencepubco.com/index.php/IJET
Research paper

\title{
Energy efficiency hybrid power management of electric vehicle (EV) charging through photovoltaic (PV) and micro grid (MG)
}

\author{
B.Rubini $^{1}{ }^{*}$, R.Krishnakumar ${ }^{1}$ \\ ${ }^{1}$ Assistant professor, Vels Institute of Science and Technology and Advanced Studies Chennai, India \\ *Corresponding authorE-mail: Rubini77@gmail.com
}

\begin{abstract}
To Analyze wireless charging system of solar PV, panel concept with electric vehicle (EV). In this developed type is a combination of solar photovoltaic (PV) and Micro grid. In this type, designed two type of operation initially the EV Charging with micro grid input supply and PV Panel. If any fault occur or grid power failure the EV battery system charge through PV panel ultimately the battery sys-tem always on line charging either grid source or PV arrays panel. The proposed methods are integrated with bidirectional converter back-toback converter with grid source. This paper focus into the power enhancement depends upon the irradiation of solar panels, so that the conversion of electrical energy is in accordance with the needs of electric vehicle attain maximum as possible. The design type of EV wireless charging methodology analyzed using MATLAB/Simulink is to get the effective power enhancement and charging to the electric vehicle continuous on line charging system.
\end{abstract}

Keywords: Electrical Battery Charging System; Power Enhancement; PV Panels; on Board Charger (OBC); Applications.

\section{Introduction}

In current decades, the fuel utilization of the conveyance sector has increased by a higher amount than any other sector. The requirement for fuel has increased day by day increased vehicles power-driven by conventional internal combustion engines (ICEs) in recent technological development typing hybrid electric vehicles are designed and powered using bidirectional converter back to back converter. Although fossil fuel resources are decreased and inadequate in nature. Various environmental issues, such as the vehicle emissions are directly related to the greenhouse effect, the technology of Electric vehicle (EV) is familiar by many countries as a new innovation effort to type of rate of harmful greenhouse gas emissions. (BEVs) Battery-powered electric vehicles close to an ultimate solution to promise with the energy crisis and global warming since they have no fuel utilization and no emissions. An active power that generates individual unit contingent on the required power demand, instead of the passive system depending on their characteristics in which the sources produce the power. Under the Energy management system (EMS) calculates each source of active management from the power output. The efficiency of energy protection and the utilization of the power system under less than an active method are not as improved compared to a passive method. Does not need any controllers or power converter in the passive method [1]. In this figure: type 1 describe low voltage DC-DC converter with filter configuration implemented and capacitance will be used for shunt connection based switch connection for power factor correction method. In this paper, we have analyzed power factor correction, Energy management, and voltage profile also can be improved across the thyristor switch, each and everyone switches connected by capacitance to improve the stability of the voltage profile and reduce the switching losses.
A capacitor or condenser is a device for storing a large quantity of electrical energy. Though the capacity of a conductor is limited to hold a charge at a particular potential, it can be increased naturally. Thus any suitable arrangement for improving the capacity of a conductor naturally is called a capacitor.

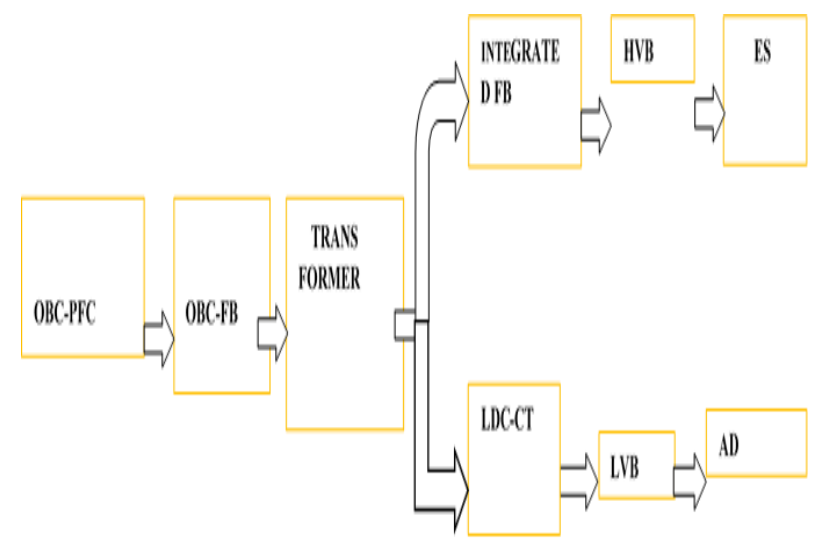

Fig. 1: Block Diagram of OLPC.

The previously used type of conventional system is broadly used in small Unmanned Vehicle's, while hybrid electric vehicles (HEVs) terrestrial-based is generally employed. Energy efficiency and fewer concerns with decreasing capacity than the aircraft imply the active method. Energy supplies are used for hybrid electric vehicles (HEVs) or Digital control power supplies of electric vehicles (EVs), the fact is excess of power and power supply are improving in efficiency is more effective. In future is predicted that energy storage systems and HEVs or EVs in-vehicle batteries will share power as a requirement or contribute interconnected 
with grid power to peak cuts or peak shifts of power consumption in the coming years of smart grids. In such a part, the important role will play a series bidirectional DC-DC converters [1-6]. Additionally, DC-DC converters can be used for charge/discharge management of rechargeable batteries but for various types like including automated guided vehicles (AGVs) of industrial equipment elevators, cranes. Such industrial equipment is started and stopped very frequently; therefore, rechargeable batteries energy can be reused in without being wasted by storing the regenerative energy generated during braking of the motors and supplying the stored power at the start-up of the motor when a large amount of power is required, thereby power conservation is stimulating. Energy supply technologies have made great improvements in the past 10 years. It is predictable that power supplies of the latest type compared to older type can reduce approximate emissions in one year total of $30,000 \mathrm{t}$ of $\mathrm{CO}_{2}$.

These new power supply products have been developed including material technologies such as ferrite Materials, by combining accumulated core technologies, transformer technologies \& circuit technologies. With these innovative products, to develop additional contributions to power conservation and the period of smart grids and microgrids in $\mathrm{CO}_{2}$ reduction. Micro-grid an interface by means of configurations at different levels delivers to the interconnection of various DGs, which is the most efficient way for the operation of DG. On the other hand, the smart distribution system makes successfully managing the micro-grids possible to realize flexible access to DG and safe, reliable, and optimal operation of the entire power system. The microgrid storage to increase the reliability and efficiency of the system employ EVs as electricity. Electrical vehicles with or without discharge capability show the optimal scheduling of the enactment to improve power, voltage, and current of the simulation results. The optimum scheduling is more sensitive to the renewable energy generation, a variation of load, load characteristics. [4].

To evaluate the active wireless EV charger the switch-on process of deliberates the initial condition switch is on before current switching is started EV charging type 1 based on the developed active wireless system calculate switch-off processes with an adequate accuracy. Finally, switching position effect indicates that 'on' or 'off' with the appropriate switching position where switchon condition the load coil have long adequate distance and the switch-on path using a gap between them and reduce EMF and extra power losses with the active path will be helpful before switching. In this paper, we optimize the power management to Electrical vehicles and power systems. In such a way that it supplies the maximum power charging without Experiencing a critical load, limitation in simultaneous type. The neutral of our work is to protect suitably long backup duration for a residence to survive with grid failure, including long-term outages that may last several weeks, by efficiently utilizing PVs and grid power.

\section{Proposed on load power conditioning sys- tem (OLPC)}

In different types of different consideration requires in the bidirectional DC-DC converter the power flow involves the smooth transition control. We have two operating types in the present case. In both the type requires the power flow:

1. For speed tracking of wide range depends on output voltage regulation 2. Current regulation with almost constant voltage during a Wide range of regenerative, active braking for buck operation.

On-Board Charger(OBC) including DC to DC converter working as a single bridge type 1 circuit including an (HFTR) highfrequency transformer to require $\mathrm{HVB}$ of a wide output voltage range. The DC-DC part includes HFTR with LDC only to accomplish a step-down ratio with $\mathrm{OBC}$ and isolation. OBC and LDC merged with HFTR can be combined to give a multi-winding structure.
In the operating moralities of the integrated structure Type 1 and Type 3 circuits are similar. The OBC voltage is produced on the distributed side both sections of the output consider the LDC and OBC through HFTR with the coupled multi-winding transformer [7], [8]. Thus, the basic structure of the OLPC Operated simultaneously by Type 1 and Type 3 circuits, (Type 1 circuit) working in the OBC operation, the secondary side voltage LDC induced by the multi-winding HFTR. Hence, the independent Type 1 from Type 3 circuits is needed as a solution for differentiating. Moreover, Type 2 Circuit the capacitor DC link connected to an induced voltage of the OBC charges on the primary side full bridge with the figure of diode MOSFET.

Though, the DC link voltage magnitude is within the voltage limit of the HVB normal range and unsettled the turn ratio operation of the OBC. Moreover, the compensation circuit is not necessary during Type 2 circuit, the diode of the PFC bias across output is reversed because the PFC part is deactivated.

The power management needs energy charging, discharging type used in electric vehicles. Initially battery power must be utilized by motor starting condition or during acceleration. The voltage and current regulation in both the types actually control the power. Considering the fixed load condition motor current should be constant and the voltage control also should maintain the power flow. Implementation of DC/DC converter of antiparallel connection implemented by the bidirectional switch is sufficient to make up for buck or boost converter. In the automobile applications, isolation between the two buses are not required and voltage conversion ratio of input and output is quite small one-third of values obtained.

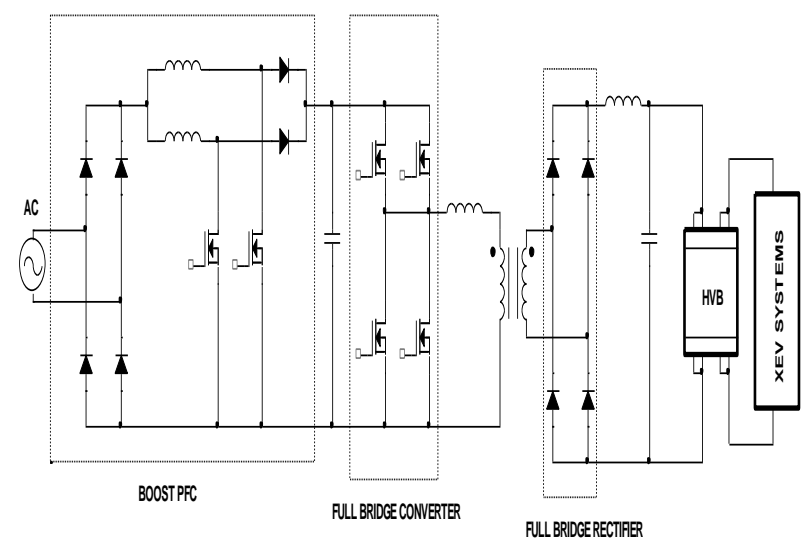

Fig. 2: Conventional OBC Standalone Type (Type 1).

The operation of converter section DC- DC Bidirectional converter has two types, continuous conduction type and discontinuous conduction type. Continuous Conduction types Performance in different ways affects its efficiency. Consequently the design of the circuit parameters are necessary for converter's operation analysis in three types (boost, buck, and buck-boost). As to provide the proper drive to the motor is always positive $\mathrm{I}_{\mathrm{L}}$ In the boost type of the converter operates to boost up the battery voltage i.e. $\mathrm{I}_{\mathrm{L}}>0$. During the operation of discontinuous conduction type the inductor across the voltage over one time period should be zero. The continuous conduction type (CCM), Bidirectional DCDC converter are generally operated in this mode and requires its design a larger valued filter inductor. Dimensions of the inductor can increase result which is not desirable. The transient response of the converter also slow down by this large filter inductor slow down any type adopt permanently the outward. With the circuit the inductor value considerably reduces operating in the discontinuous conduction type (DCM).

The inductor value can reduce it to get the operation response of DCM is faster and also the converter design by operating in DCM type to have a high power density. During DCM operation turn 'ON' losses is zero, reverse recovery loss is low for diode. But, the operation of the DCM main switch is in off condition at that time the load current increase twice, turn on condition loss will 
increases. the switch with output Capacitance of the inductor tries to oscillate The switch is turn on condition will acquire freeloading of the inductor current exhibits in the meantime and hence causes power dissipation and electrical stresses on the devices. During negative effects of the DCM operation major disadvantage associated the efficiency reduces all this the DCM operation.

The remedial measures taken as well as for the freeloading ringing have to be ensured by the soft switching techniques. Therefore, the configuration of the characteristics in order to improve the switch involved to the LDC on the secondary side the centre-tapped rectifier is used for delaying convinced voltage, the blocking switch is fully turned off functionally and the LDC from the CPU in Type 1 detaches the secondary side.

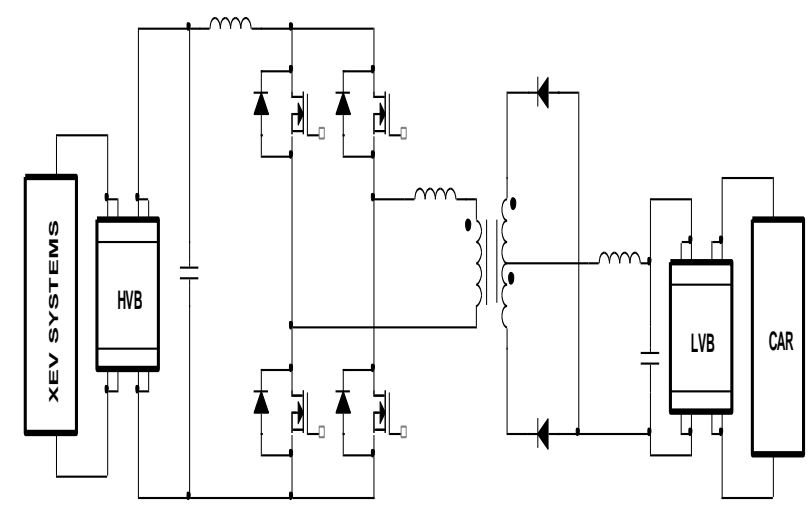

Fig. 3: LDC Standalone Type (Type 2).

In Type [2], fully turn-on the LDC operation the switch is tolerates. In accumulation, the real-time charge in Type 3 the PWM switch efficiently implements. Every type of the OLPC the status of the group is determined and classified as driving or charging; activate the Type 1 and Type 3 while the charge is stopped by charging through the grid power. In Type 2 is working condition of the vehicle is driven supported Based on (BMS) the battery management system, OLPC charges simultaneously the HVB firs charges and after LVB starts to charge in Type 3. Charging condition is the state of LVB is called state of Charge (SOC) \& internal electrical devices are used at the charging period. Once the vehicle is stopped and focused by (SOC) the stage of charge Type 1 and Type 3 circuits.

\subsection{Operating types of on load power conditioning sys- tem}

OLPC Proposed method operates in the conventional OBC and LDC, instead of OBC standalone type same as respectively, (Type 3) simultaneous mode during the operation of the OBC, which can be, charge the HVB as shown in Fig 3 above.

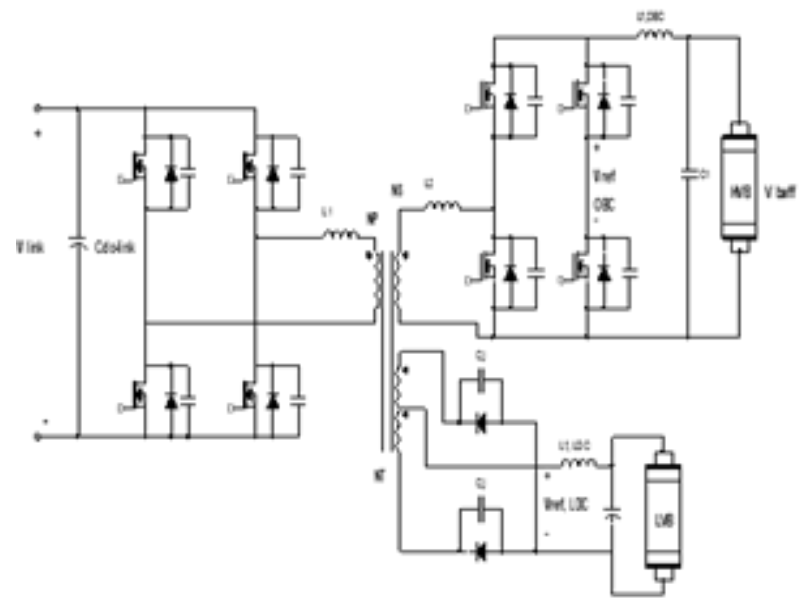

Fig. 4: Design of Proposed OLPC with Built in Buck.

\subsubsection{Design consideration of proposed OLPC}

The PFC design is similar to conventional process of the OBC, HVB could satisfy the output voltage can be varied with the wide voltage range. The design of PSFB conventional converter also same as DC-DC converter from secondary side the multi-winding HFTR should be considered and the parasitic capacitive components connected across the switches as shown in Fig.4 [1].Moreover, the induced voltage to control in the multi-winding HFTR a compensation circuit is proposed ensure as well as steady charging performance of the LVB in Type 3.

\subsubsection{ZVS inductor design of OLPC}

The switch can be realized in ZVS condition by a resonant capacitor or by the switch parasitic capacitance in parallel across the controlled semiconductor switch $\mathrm{CR}$ is added. The conventional PSFB as the proposal of the ZVS inductor OLPC is nearly identical. The proper design consideration is needed for ZVS inductor because it will connected to multi winding structure.

In Type 1, the proper design method of conventional the DC-DC converter with inductor ZVS is designed, because of the LDC side secondary is detached by the delaying switch. In Type 2 operation practical no load condition OBC primary side is not resonance reproduced fully charging without current flow the DC link of the Power Factor Correction. Therefore, the full bridge circuit transient state consider the MOSFETs switches are the LDC prime using the conventional converter in the Type 2 operation. The prime side of the LDC consider only the proposal of the inductor and the secondary side of conventional LDC in the Capacitance and inductance values are increased in the multi-winding structure.

Although the circuit elements and the parasitic capacitance components are newly added in Type 3, Type 1 in the proposal typically over a period of time lagging leg transition of the ZVS inductor is same as the design criterion. For unchanging operation in Type 1, Type 2, and Type 3 can be resolved through this process the least inductance of the ZVS inductors. The design specification of the duty loss $(\delta D)$ is fixed based on the margin design value of the ZVS inductor. Thus, the design consideration is implemented for the ZVS inductors concerned with the maximum load condition.

\subsubsection{Design of OLPC with HFTR}

The Design of OLPC proposed HFTR is two operating working conditions, step-up and step-down operation and connected with OBC and LDC Charges, the ratio of Ns to NT HFTR priority task effectively "inverting" the relative priorities of the two tasks in terms of the design of proposed OLPC. At first turn in the winding design process of NT is fixed for the reason that the current through the winding NT is high in Type 2 operation [7]. When 1 turn NT is fixed at secondary turns are maximum can be determined, operating condition of the LDC the L, C this are resonant parameters reflected in the ZVS condition. The HFTR primary, secondary turn ratio aspects in the $\mathrm{OBC}$ operation can be signed by using the design of secondary turns.

The LDC side Ns/NT of turn ratio is calculated using the expansion in [1]-[5]. The ratio of $\mathrm{Ns}_{\mathrm{s}}$ to $\mathrm{N}_{\mathrm{T}}$ is designed with high rating (400 V DC) input and lower rating (13 V DC) output at the huge step-down ratio condition. Because the HVB, LVB voltage ranges are 250 to $400 \mathrm{~V} \mathrm{DC} \& 13$ to $15 \mathrm{VDC}$ input source, the LDCvaries normally. By way of the ZVS condition in these input and output settings. In the operation of step-down process in LDC of the maximum NT turns are decreases and ratio of primary and secondary turns ratio will increases with multi winding HFTR in ideal position. The turn ratio of the multi-winding HFTR ideal position, NP: NS: NT is 11:15:1. As a result, at the inconsequential input voltage of $380 \mathrm{~V} \mathrm{DC}$.

However, the voltage V DC control it is difficult to satisfy in this case and voltage ratio, which varies from 250 to 400 when the inconsequential of the DC-DC part input voltage is $380 \mathrm{~V} \mathrm{DC}$ in the OBC output range of the HVB. In addition, the change the 
position part of sequence of events first and second sides of the OBC has ratio between the small turn ratios. Exponentially stepup condition primary to secondary turns will increases $\delta \mathrm{D}$ also increases as the turn ratio of the OBC. Voltage controlled by OBC with DC link which does not interrupt to vary from 350 to $410 \mathrm{~V}$ $\mathrm{DC}$, to satisfy the conditions to control enactment of the PFC. Design flexibility of this variable control DC link the turns ratio through accountable of the $\mathrm{OBC}$ on the output voltage can be protected. Consequently, function of the OBC the enactment of the suggested OLPC to optimize the control voltage output is calculated to vary according to the output and based on the variable range Deff is calculated again.

The voltage condition furthermore in the basic design procedure considered for variable DC link is designed again input and output circumstances of the OBC part and in each condition, as exposed in Fig.5, That time, the design of the upper limit is by considering the dead time and $D_{\text {eff }}$ and $D_{\max }=0.97$. Thus, the designed with this limitation should be duty cycle. Conditions with the variable all to fulfil input and output condition. Based on the calculation of the results, design a transformer it is valid with a turn $\mathrm{PFC}$ variable output control. In this paper, control voltage of the PFC of the primary variable output is used for the output voltages with the extensive device which operates intermittently rather than continuously. Therefore, Np: Ns: Nt, is 13:15:1 and the final designed turn ratio.

\subsubsection{To control convinced voltage of additional circuit}

A circuit-integrated buck that magnitude of the convinced voltage freely controls using the enhanced conservative regulator through the blocking switch with the execution of the separated Type1 is furthermore caused by the multi-winding HFTR proposed method to solve the induced voltage. An arrangement similar to the conventional buck converter is the proposed circuit, which uses on the LDC as the buck inductor of the secondary side and addition of a freewheeling diode, MOSFET of the output filter. Inductor of the centre-tapped rectifier as shown in Fig.5. Induced voltage.

To operation in each, type LVB through the different suitable level for charging. Moreover, change in the switching status through the operation of conventional LDC is also executed in Type. In Type 1, from the multi-winding HFTR complete the centre-tapped rectifier MOSFET switch is turned off and blocks the distribution of the convinced voltage of the LDC on the secondary side to the LVB. The LDC operation in Type 2 with this switching status, acting as the line-without manipulating the independent operation of continuous converter in turn-on condition. At this time, MOSFET in turn on position occur in additional conduction losses, but than those caused in the conservative post regulator the losses are much smaller. In Type 3, the output voltage in the direction of the LVB through the self-governing controller, the MOSFEET controls in a related method to the conservative switching device. In this operation, the role of the buck inductor performs the output filter inductor.

the primary side on the circuit operation of the $\mathrm{OBC}$ from the powering period depending duty analysis and the period $\mathrm{t} 2-\mathrm{t} 4$ is the duty loss $(\delta \mathrm{D})$ in which the power is not delivered from the primary side to the secondary side because of the slope in the decreasing/increasing current which is the power actually delivered to the output and also detailed on to the switching period of the integrated buck is required for the characteristics analysis of the duty period (1-D).

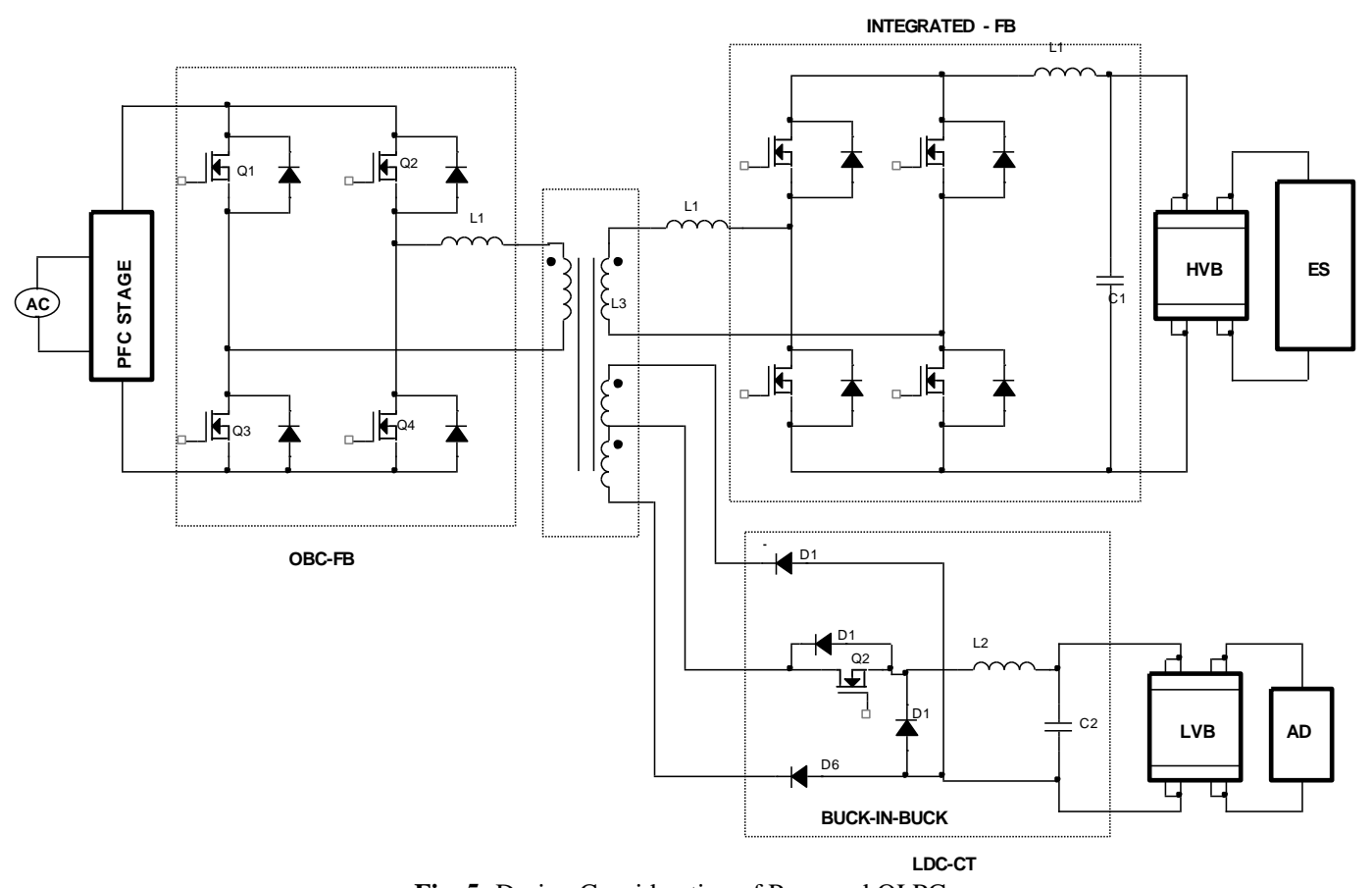

Fig. 5: Design Consideration of Proposed OLPC.

When for the integrated buck reasonable control method is considered according to the simulation result rising carrier synchronization will be analysed.

Proposed OLPC can be rebuilt a full bridge circuits independent operation and multi winding replace two HFTR in the conventional system. In the OLPC is the conventional OBC and LDC compared with the volume of the HFTR and the no.of major components. The total magnetic core transformer are increased. Size of the circuit components and the heat sink can be reduced seriously. The high power conductors and insulation between the LDC and HVB can be eliminated. Design installation structure can be simplified and improved. The structure leads clear Installation to up- grading of the cooling system shared and an increased capacity. OBC-LDC simultaneous type both the HVB \& LVB that can be charge at the similar time through the multi winding HFTR.

\section{OBC-LDC synchronized type}

The OBC work as (INTEGRATED-FB) integrated full bridge rectifier on the secondary side and the LDC of the centre-tapped rectifier secondary side is operated to charge the LVB. In this OBC-LDC type stress in primary side not applied on PFC part FB. HVB \& LVB of the Total charging power should not beat the 
limit $3.3 \mathrm{KW}$. The standalone type max output, from the extreme power of charging the OBC\& the charging power of the LVB is Distributed and it is delivered by the PFC and the DC-DC of prime side in the OBC [9]. A trade-off design of optimization for the snubber capacitor exists. An experiments of series were conducted to the capacitance determined the switching loss based on condition for minimum overall device.

\section{LC filter design}

To improve the filtering action used a capacitor -inductor filter is rectified voltage and current. The inductor or capacitor alone the filter action cannot perform satisfactorily for low power applications as earlier is suitable and the latter is suitable for high Power applications. Though, if both the inductor and capacitor are combined, dc voltage and current they produce high quality. The inductor is used to smooth out current in the variations while the function of the capacitor is to smooth out in the voltage variations. Uniformly the flow of current, the inductor capacitor filter is used. Widely full-wave bridge rectifier in high-power applications with an Inductor- Capacitor filter implemented. From previous sections we know that, if used alone, a $207.898 \mathrm{MH}$ inductor is needed to reduce the ripple to $3 \%$. Similarly, If used alone, a $10.238 \mathrm{mF}$ capacitor is needed to reduce the ripple to $3 \%$ in the circuit under consideration $[10,11]$.

The aim of this section is to find out the value of an inductor and capacitor used together in the way to reduce the ripple to $3 \%$ shown in Fig 4. To design the filter is to select the approach taken when used alone and then vary the value of inductor until a $3 \%$
Ripple is obtained. Arbitrary value of capacitor; about $25 \%$ of the value that was needed to reduce the ripple to $3 \%$.

Using this value the voltage and current waveforms are obtained from ATP .The trial and error using values are obtained by the verified ATP type 1. ripple Current in percentage as a function of inductor in $\mathrm{mH} 3 \%$ current ripple is obtained when a capacitor of $2.5 \mathrm{mF}$ and an inductor of $12.5 \mathrm{mH}$ are used.

Ripple percentage $=(\mathrm{Irms} / \mathrm{Io}) \times 100=3 \%$

Ripple percentage $=(\mathrm{Vrms} / \mathrm{Vo}) \times 100=3 \%$

Vrms of ripple using voltage waveform $=0.707 \times$ Vp Volts.

Irms of ripple using current waveform $=0.707 \mathrm{x}$ Ip

\section{Matlab simulink type}

Solar panel is connected converter and Battery SOC, voltage, current waveforms confirm the accuracy of the proposed method. Storage part. The proposed concept in this paper is based on mathematical approach to formulate the energy management of battery, OBC-LDC charging section. Design and calculated values are used and the same are developed. The developed type 1 Integrated with DC-DC bidirectional converter section. In this approach, developed in MAT LAB/SIMULINK type 1 . The type 1 consists of the following main sections. They are three phase input ac power type 1, power circuit, integrated-FB and LDC-CT type 1 .

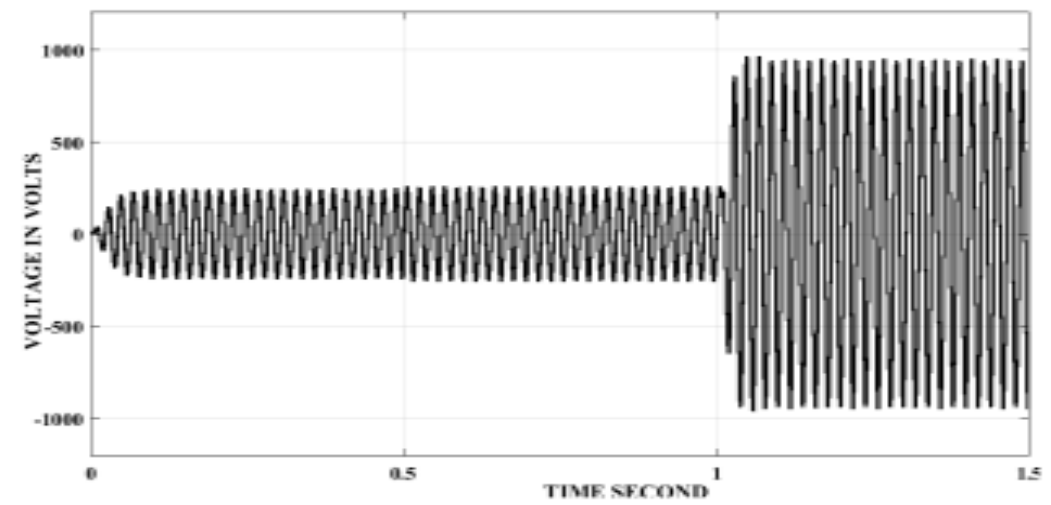

Fig. 8: Low Voltage Battery Measurements.

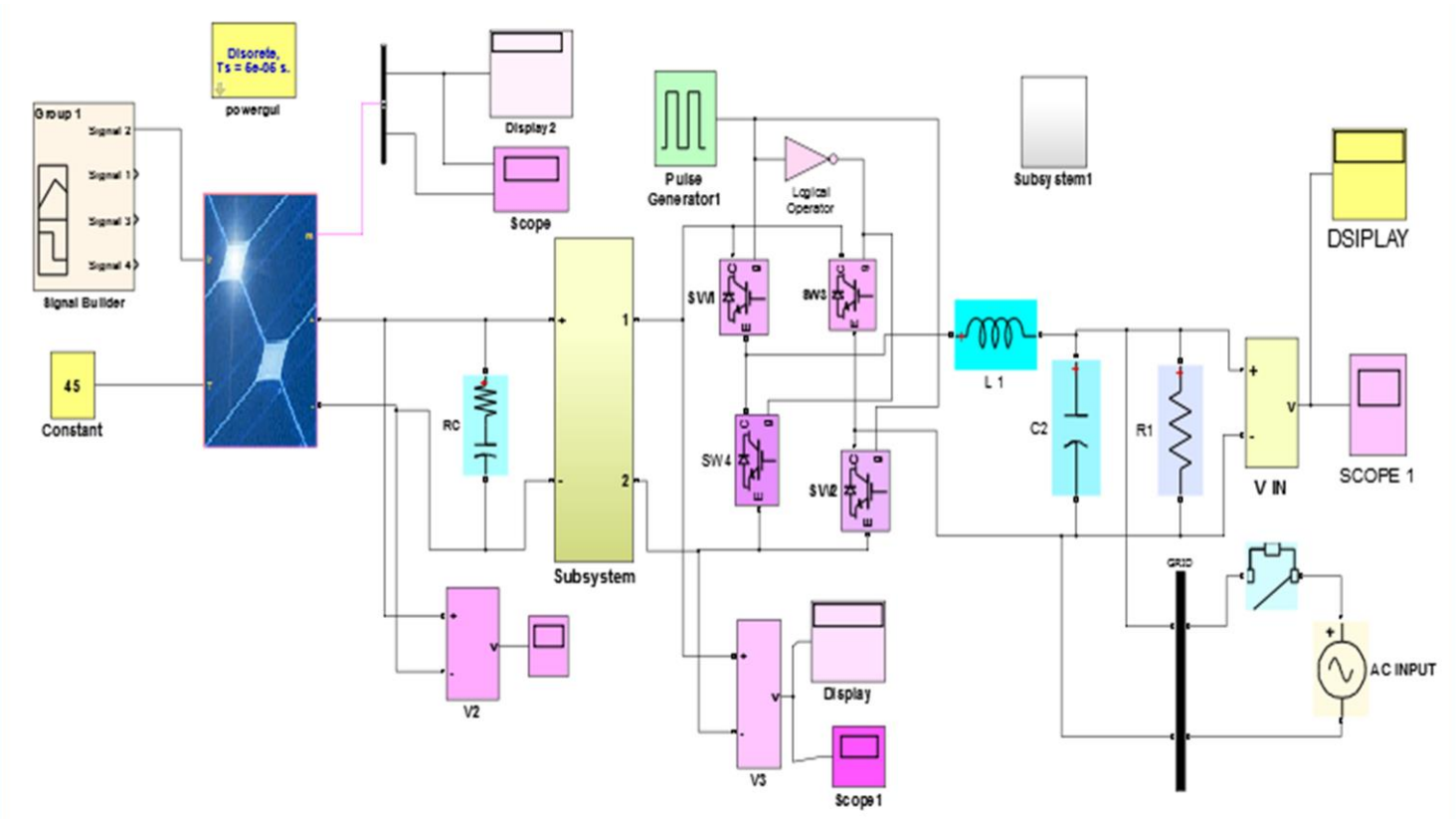

Fig. 6: Solar Panel Connected to Ev Charging System. 


\section{Result and discussion}

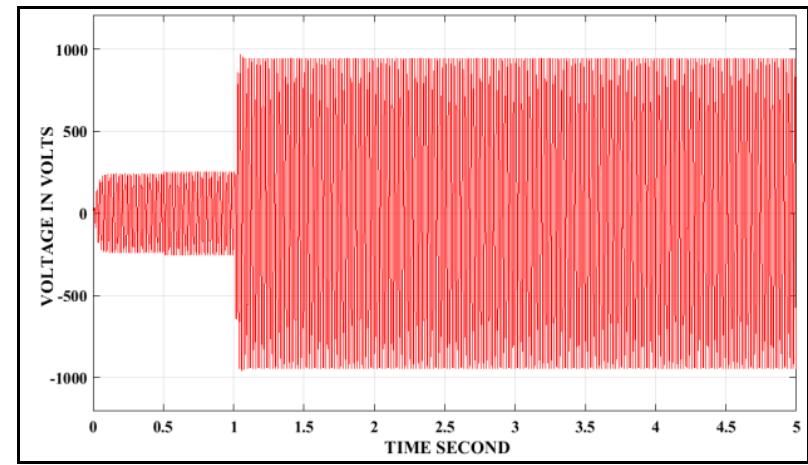

Fig. 7: High Voltage Battery Measurements.

Output voltage suitable filter circuit are added and suppressed the reflected over shoot voltage near the terminal. The amplitude of motor terminal voltage is maintained nearly equal to input supply voltage. The frequencies of the output voltage are matching the switching Frequency. Filter circuit added at the load terminal which minimized harmonics at a feasible limit and the reflected voltage can also be eliminated. So that a pure fundamental output is supplied from the Main circuit. Figures (7) and (8) shows the HVB and LVB output voltage.

\section{Solar PV Panel Output Voltage and Current}
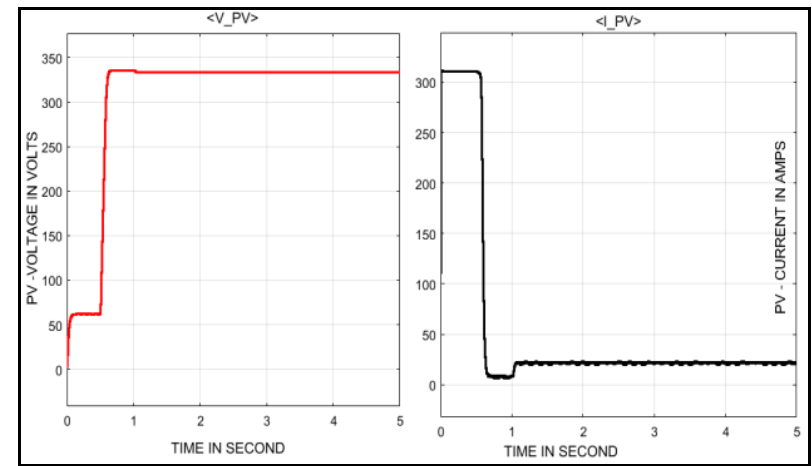

Fig. 9: Shows the Battery Charging Period.

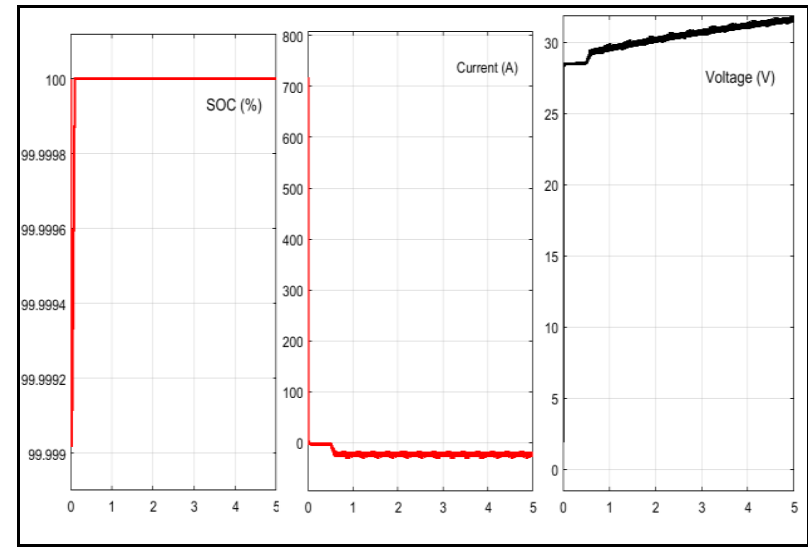

Fig. 10: Battery Charging V,I,Soc.

SOC, voltage, current waveforms confirm the accuracy of the proposed method.

\section{Conclusion}

The micro grid integrated over bidirectional back-to-back converters are linked to the appropriateness power management system. The power requirement in the micro grid is shared by the PV panel the PV array type 1 is proportional to their solar irradiation. Scheme based on the different in dc link voltage proposed control level due to the irradiation of the sun, is simple and unique. From the utility with a kind of demand management to increase the energy efficiency with least amount of energy used by PEVs charging included energy management algorithm facilitates. In case of high power demand in the charging type are combined with micro grid and PV array, in this AC source off type the EV battery charged through PV panel only during sun rays not present or night period the EV charge through micro grid as a input source. The power management utility intended through back-to-back bidirectional converters. Though the PV panel and micro grid are totally isolated through wireless transformer. Proper switching of the breaker and other power electronics switches has been proposed during synchronization process. Also, they can be extended to power management up to the level of maximum possible EV charging is admirable with other existing technique. System design included with this power management to avoid elaboration complex EV charging methodology.

\section{References}

[1] Bohwa lee sejin kwon, Poomin park keunbae kim "Active Power Management System for an Unmanned Aerial Vehicle Powered by Solar Cells, a Fuel Cell, and Batteries" IEEE Transactions on Aerospace and Electronic Systems Vol. 50, No. 4 October 2014.

[2] Liu J and Peng H "Typing and control of a power-split hybrid vehicle" IEEE Transactions on Control Systems Technology 2008 PP 1242-1251.

[3] Thou thong P. Real S and Davat B “ Energy management of fuel cell/battery super capacitor hybrid power source for vehicle application" Journal of Power Sources 2009 pp 376-385.

[4] Mosaddek Hossain Kamal Tushar, Chadi Assi, Martin Maier, and Mohammad Faisal Uddin "Smart Microgrids: Optimal Joint Scheduling for Electric Vehicles and Home Appliances" IEEE Transactions on Smart Grid, Vol. 5, No. 1, January 2014

[5] Yanjie Guo, Lifang Wang, Qingwei Zhu, Chenglin Liao, and Fang Li "Switch-On Typeing and Analysis of Dynamic Wireless Charging System Used for Electric Vehicles" IEEE Transactions on Industrial Electronics, Vol. 63, No. 10, October 2016.

[6] Karen L. Butler, Mehrdad Ehsani, Preyas Kamath, "A MatlabBased Typeing and Simulation Package for Electric and Hybrid Electric Vehicle Design" IEEE Transactions on Vehicular Technology, Vol. 48, No. 6, November 1999.

[7] K. Qian, C. Zhou, M. Allan, and Y. Yuan, "Typeing of load demand due to EV battery charging in distribution systems," IEEE Trans. Power. Syst., vol. 26, no. 2, pp. 802-810, May 2011.

[8] P. Zhang, K. Qian, C. Zhou, B. G. Stewart, and D. M. Hepburn, "A methodology for optimization of power systems demand due to electric vehicle charging load," IEEE Trans. Power. Syst., vol. 27, no. 3, pp. 1628-1636, Aug. 2012.

[9] K. Clement-Nyns, E. Haesen, and J. Driesen, "The impact of charging plug-in hybrid electric vehicles on a residential distribution grid," IEEE Trans. Power Syst., vol. 25, no. 1, pp. 371-380, Feb.2010.

[10] N. Shanmugasundaram, S. Thangavel, "Typeling and Simulation Analysis of Power Cable a Three Level Inverter Fed Induction Motor Drive". Journal of Computational and Theoretical Nano science. Vol. 14, 972-978, 2017.

[11] N. Shanmugasundaram, R. Vajubunnisa Begum, "Typeling and Simulation Analysis of Power Cables for a Matrix Converter Fed Induction Motor Drive (MCIMD)" Jour of Adv. Research in Dynamical \& Control Systems, 11-Special Issue, November 2017. 